\title{
Adaptive optics concept for multi-object 3D spectroscopy on ELTs
}

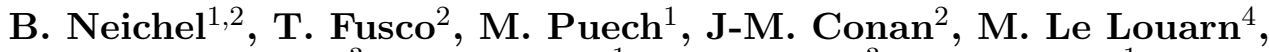 \\ E. Gendron ${ }^{3}$, F. Hammer ${ }^{1}$, G. Rousset ${ }^{3}$, P. Jagourel ${ }^{1}$, \\ and P. Bouchet ${ }^{1}$ \\ ${ }^{1}$ GEPI, Observatoire de Paris-Meudon, 5 Place Jules Janssen 92195 Meudon, France \\ email : benoit.neichel@obspm.fr \\ ${ }^{2}$ ONERA, BP 72, 92322 Chatillon Cedex, France \\ ${ }^{3}$ LESIA, Observatoire de Paris, 5 Place Jules Janssen 92195 Meudon, France \\ ${ }^{4}$ ESO-European Southern Observatory, Karl-Schwarzschild-Straße 2, \\ Garching, D-85748 Germany
}

\begin{abstract}
In this paper, we present a first comparison of different Adaptive Optics (AO) concepts to reach a given scientific specification for 3D spectroscopy on Extremely Large Telescope (ELT). We consider that a range of $30 \%-50 \%$ of Ensquarred Energy (EE) in H band $(1.65 \mu \mathrm{m})$ and in an aperture size from 25 to 100 mas is representative of the scientific requirements. From these preliminary choices, different kinds of AO concepts are investigated : Ground Layer Adaptive Optics (GLAO), Multi-Object AO (MOAO) and Laser Guide Stars AO (LGS). Using Fourier based simulations we study the performance of these AO systems depending on the telescope diameter.
\end{abstract}

Keywords. instrumentation: adaptive optics — techniques: 3D spectroscopy — galaxies: highredshift

\section{Introduction}

The new era of astronomical telescopes with diameters reaching 30 to $100 \mathrm{~m}$ will provide a dramatic advance in our understanding of the universe. The concept of ELTs is unique to complement the detection which will be made from Space. By accommodating high spectral resolution, 3D spectroscopy devices and relatively large fields of view (10 arc minutes), ELTs will be a privileged tool for the study of formation and evolution of galaxies.

Adaptive Optics enables large telescopes to provide diffraction limited images by real time correction of turbulence. Because the contamination by the interstellar medium light is one of the main issue in extragalactic studies it is required to observe in a direction far from our galactic plane. In that case, the density of stars becomes dramatically small, and because of anisoplanatism effects, classical AO working on Natural Guide Star (NGS) can not be used. To overcome this problem, new AO techniques (GLAO, MOAO) have emerged in the last few years to increase the corrected field using NGS. These new methods are based on a full measurement of the 3D turbulent volume using several NGS. On the other hand and to improve sky coverage, Laser Guide Stars (LGS) have been proposed (Foy et al. 1985, Lelouarn et al. 1998). Should some critical issues be solved (cone effect, spot elongation, tilt indetermination) this last solution would allow the ultimate scientific requirements to be met.

In section 2 we present the specifications required to achieve scientific goals imposed by astrophysical studies of high-redshift galaxies. Section 3 is then dedicated to a first 
performance evaluation for AO systems, and some preliminary results from Fourier based simulations will be shown.

\section{Extragalactic requirements for ELT}

One of the main science cases for ELTs is the physics of galaxies at very high redshift (Hook 2004). Extragalactic studies will benefit from the large capabilities of ELTs in light concentration and spatial resolution: $3 \mathrm{D}$ spectroscopy of galaxy up to $m_{A B}=26-27$ is expected. Such data will have a large impact on our understanding of the assembly of dark and visible matter (from $z=0$ to $z=5$ ), the physics of galaxies near the reionisation $(z=6-9)$ and the search for the primordial galaxies $(z \gg 6)$.

3D spectroscopy of distant galaxies will necessitate to obtain spatially resolved spectra of very faint and physically small objects.

From a spectroscopic point of view, it will be needed to resolve velocities of a few tenths of kilometers, and to avoid contamination between atmospheric $\mathrm{OH}$ lines. This implies to use a spectral resolution $R>5000-10000$.

From a spatial point of view, if we consider a typical galaxy redshifted at $z=6$, its size (half-light radius $R_{\text {half }}$ ) will be around $R_{\text {half }}=0.15$ arcsec (Bouwens et al. 2004). Preliminary results (Puech et al. 2005) from GIRAFFE (3D spectrograph at the ESO-VLT) show that, in dynamically unrelaxed systems, the gas is far more extended than the stars: a pixel size around $R_{\text {half }}$ can be sufficient to study galaxy dynamics. Thus, to be able to resolve different areas (HII regions, SN, ...) and correctly sample high redshifted galaxies, the spatial resolution element has to be between 25 to 100mas. In addition, a large FoV will be required to avoid cosmic variance and obtain a statistically unbiased sample. The scientific goal would require a multiplex factor ranging from 10 to 100 objects, which implies a total FoV of a least 5 arcmin in diameter. Only new AO concepts could offer such an angular resolution over such a wide field.

Finally, in a first approach, Assemat (2004) shows that a light coupling of $30-50 \%$ (in a spatial element of resolution of 250 mas, $\mathrm{H}$ band) is required to detect the $H \alpha$ line with sufficient SNR (greater than 3), in a reasonable exposure time and for $8 \mathrm{~m}$ class telescopes. In first approximation we will preserve this range of light coupling. Once more, AO is essential to increase the concentration of light within an even smaller spatial element of resolution and meet the scientific requirements.

In the following study, we have fixed the scientific specifications at $40 \%$ of light coupling ( $\mathrm{H}$ band) in a resolution element of 25 or 50 mas. This could be subject to future adjustments, but seems to be a reasonable work baseline.

\section{Investigating AO solutions}

\subsection{Description of $A O$ systems}

- The objective of GLAO is to provide a wide and uniformly corrected field, but with only very partial correction (Hubin et al. 2006, Rigaut 2001). This can be performed by compensating for the boundary layer of the atmosphere, which is in the same time the location of most atmospheric turbulence and the layer for which correction remains valid on a wide FoV. Several NGS (and several Wave Front Sensors (WFS)) have to be used to extract the boundary layer signal from the whole turbulence volume. This can be done using a simple average of data coming from all the NGS. Then, only one Deformable Mirror (DM), usually conjugated to the telescope pupil, is used to correct this ground layer and obtain a uniform correction in the FoV. 
We have studied here the performance of a GLAO correction in a total FoV of 2 arcmin in diameter. This implies that each point of the 2 arcmin FoV sees the same correction (see sect. 3.3 for results).

- Instead of compensating the whole field, MOAO performs the correction locally on each scientific object (Hammer et al. 2001). Several off-axis NGS (a constellation), even widely outside the isoplanatic angle, are considered to perform a tomographic measurement of all the turbulence volume around each scientific object (i.e. direction of interest). The optimal correction is then deduced from the turbulence volume estimation and applied using a single DM per direction of interest.

We focus this study on a MOAO system working with a NGS constellation of 2 arcmin in diameter around each scientific object (see sect. 3.3).

- To overcome sky coverage issues Laser Guide Stars could be used where the density of stars becomes too small for good turbulence analysis even with GLAO or MOAO concepts. But LGS suffers from limiting effects (Tip-Tilt indetermination, focus anisoplanatism, spot elongation) which makes their implementation difficult, especially when Large or Extremely Large Telescopes are considered. We study here the possibility of using a LGS with a partial tip-tilt correction. In this case, we suppose a LGS in the direction of interest providing a perfect correction up to the DM cut-off frequency except for tip-tilt which is corrected at different levels: correction of $80 \%, 50 \%$ or $0 \%$ (no correction) in variance. A partial correction of Tip-Tilt could be achieved if a NGS is weak or far from optical axis for complete Tip-Tilt determination. No other LGS effects (focus anisoplanatism, spot elongation) were considered. However, previous studies have shown that focus anisoplanatism can be solved for instance by using several LGS per object in order to sense the whole cylinder turbulence path (Viard et al. 2002). Concerning spot elongation issue, customized CCD could be used (Beletic 2004).

\subsection{Simulation parameters}

For GLAO and LGS modes, we have developed a simulation tool based on a Fourier approach (Jolissaint et al. 2006) which computes an AO corrected PSF for different AO systems (Conan et al. 2003). MOAO simulations were done with CIBOLA, an analytical AO modeling tool developed by B. Ellerbroek (Ellerbroek 2004).

In GLAO simulation, we assume to measure the average phase over a full 2arcmin diameter disk. The pupil layer is then fully corrected up to DM cut-off frequency. The upper layer correction cut-off is reduced as the altitude increases (and derived from the WFS FoV). LGS simulations are based on classical AO simulations (correction on-axis) with a partial tip-tilt correction. Finally, MOAO simulations assume a tomographic measurement of turbulence thanks to three stars at the edge of the FoV (2 arcmin in diameter) and a perfect correction up to DM cut-off frequency. For the three systems, the DM cut-off frequency is determined by the inter-actuator distance fixed at $0.5 \mathrm{~m}$.

Concerning the turbulence, we suppose a typical Paranal turbulence profile, discretized in 10 layers. The total seeing is $0.85 "$ at $0.5 \mu \mathrm{m}$ and outer scale of turbulence is fixed at $\mathrm{L} 0=50 \mathrm{~m}$.

Note that some limitations such as WFS noise, temporal error or aliasing are not taken into account. Results presented here have therefore to be considered as limit (and for sure optimistic) cases.

\subsection{Simulations results}

The estimated performance for the three AO systems are presented in Figure 1. We plot the Ensquarred Energy (EE) in a given resolution element (50 or 25mas) for different telescope diameters. We recall that the scientific specification considered here is to reach 
$40 \%$ of EE, which corresponds to a gain of a few orders of magnitude compared to the uncorrected case. Seeing limited case leads to EE $\sim 0.1 \%$ in a resolution element of 25 mas and $\mathrm{EE} \sim 0.7 \%$ in $50 \mathrm{mas}$.
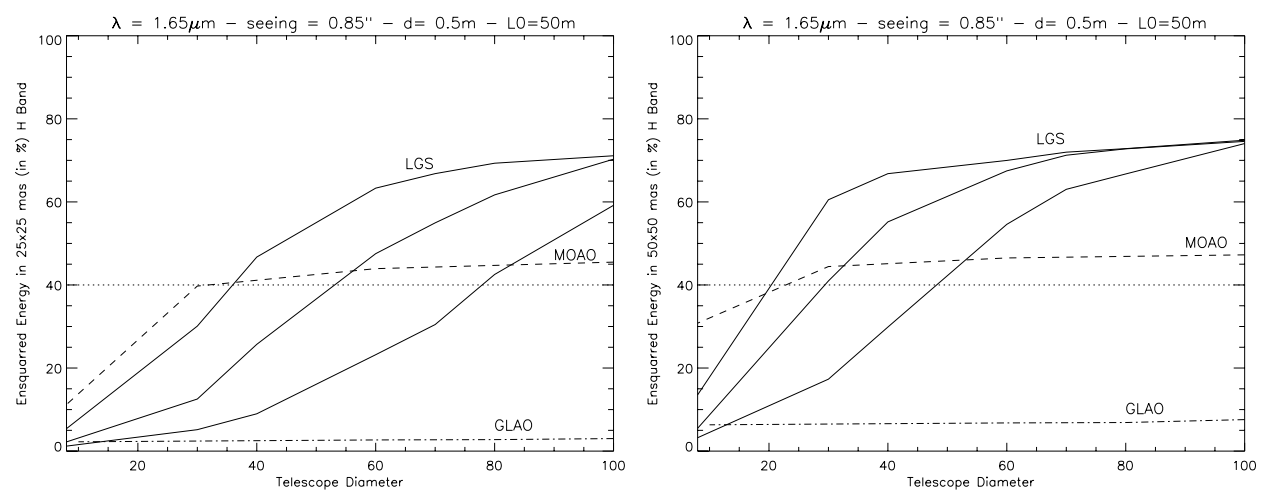

Figure 1. Effect of the telescope diameter (from 8 to $100 \mathrm{~m}$ ) and resolution element size (right $=$ $25 \mathrm{mas}$, left $=50 \mathrm{mas}$ ) on performance for three AO systems : GLAO (dashed-dot line) with total $\mathrm{FoV}=2$ arcmin, MOAO (dashed line) for constellation diameter $=2$ arcmin and LGS (solid line, from top to bottom : $80 \%-50 \%-0 \%$ Tip-tilt correction).

Simulations show that :

- Even in little field ( 2 arcmin) the GLAO based system could not reach the scientific specifications and performance stay very limited $(\mathrm{EE}<10 \%)$. GLAO provides a typical gain of 10 in EE compared to the seeing limited case, which is not consistent with the required performance.

- Scientific specifications could be reached in a MOAO concept particularly when diameter is large ( $\mathrm{D}>30 \mathrm{~m})$. Indeed, as we consider a fixed resolution element, performance benefits from diffraction effects: the size of the PSF decreases as diameter becomes larger, and so, the concentrated light in a given resolution element increases. For telescope diameter smaller than $30 \mathrm{~m}$, the PSF becomes large compared to the resolution element size with the effective correction obtained in a MOAO configuration.

- Using LGS, scientific specifications are achieved when the diameter is large enough to overcome the tip-tilt effect or if a NGS is close enough to ensure a good tip-tilt correction. Like in the MOAO case, LGS benefits from diffraction effects as telescope diameter becomes larger. For small diameters, the impact of the resolution element size is all the more critical that we consider a partial tip-tilt correction. More precisely, it seems that an important parameter could be the ratio $D / L 0$ multiplied by the resolution element size. Indeed, we see that when this parameter becomes greater than 50mas, scientific requirements are achieved even without tip-tilt correction. In other cases, using a NGS even weak or far from the optical axis for tip-tilt measurements should be sufficient to reduce the residual tip-tilt variance at acceptable levels.

\subsection{Discussion on sky coverage}

To perform a correct analysis of turbulence, MOAO needs at least three stars around the direction of interest. Depending on the position and magnitude of these stars, performance will change. On the other hand, the probability to find a suitable constellation decreases as we observe at high galactic latitudes $\left(b>45^{\circ}\right)$. It has been shown in a previous study (Assemat 2004) that a constellation of 2 arcmin in diameter (as the one used to perform the simulations) could provide $50 \%$ sky coverage at $b=30^{\circ}$ with $R=16$ 
stars (respectively $b=60^{\circ}$ with $R=17$ ). But as we consider a perfect case (no noise) for these simulations, we except much lower performance in a more realistic configuration. Sky coverage could be a critical issue for MOAO systems working with NGS.

In the LGS case we assume a partial tip-tilt correction. That implies to use a NGS for tip-tilt determination. But as we consider only a partial tip-tilt correction, the probability to find an adequate NGS is large even in fields far from the galactic plane.

If scientific specifications require an aperture size lower than 50mas and EE $>40 \%$, LGS will become mandatory for $100 \%$ sky coverage.

\section{Conclusion}

We have presented here some recent results concerning AO concepts for future ELTs and particularly for 3D spectroscopy. First numerical simulations show that even for quasi-ideal case, GLAO could not achieve the scientific goal, MOAO could be used with a limited sky coverage and only a LGS based system could offer a 100\% sky coverage. This article also shows that the implementation of rather simple LGS systems without tip-tilt correction could be of interest specifically for very large telescope diameters.

Additional simulations are mandatory to improve the science specifications and evaluate if coupling or aperture size could be relaxed. Further analysis should then lead to a detailed AO system definition.

\section{References}

Assemat, F. 2004, Apport de la haute résolution angulaire sur létude des galaxies lointaine: imagerie, optique adaptative et spectroscopie $3 D, \mathrm{PhD}$ thesis, Observatoire de Meudon.

Beletic, J-W. 2004, SPIE 5382, 706

Bouwens, R. et al. 2004, ApJ 611, L1

Conan, R. 2003, SPIE 4840, 393

Ellerbroek, B.L. 2004, SPIE 5490, 625

Foy, R. \& Labeyrie, A. 1985, A\&A A 152, L29

Hammer, F. et al. 2001, SPIE 5382, 727

Hook, I. 2004, SPIE 5489, 35

Hubin, N., et al. 2006, C. R. Physique 6, in press

Jolissaint, L., Veran, J-P. \& Conan, R. 2006, J. Opt. Soc. Am. A 23, No. 2

Lelouarn, M., Foy, R., Hubin, N. \& Tallon, M. 1998, MNRAS 295, 756

Puech, M., Hammer, F. \& Flores, H. 2005, A\&BA, submitted

Rigaut, F. 2001, in: E. Vernet, R. Ragazzoni, S. Esposito, N. Hubin (eds.), Beyond conventional adaptive optics: a conference devoted to the development of adaptive optics for extremely large telescopes, 11

Viard, E., Hubin, N. \& Lelouarn, M. 2002, SPIE, 4007, 106

\section{Discussion}

Hook: The plots showing percentage enclosed energy vs. telescope diameter, D, for different types of $\mathrm{AO}$ are interesting. However, the absolute flux in the aperture is the real specification, and telescope diameter clearly affects that. It would be useful to see the comparison presented as a function of $\mathrm{D}$ for absolute flux in a fixed aperture.

Bouchet: The curves presented here (coupling vs. telescope diameter) are useful only to qualify the AO performances (spatial resolution) and to compare different modes. It is clear that for a given curve increasing diameter would improve the efficiency. The very important point to be analyzed (which is by no way simple) is to understand how the specifications chosen (e.g. $40 \% \mathrm{EE}$ ) varies with respect to the $\mathrm{S} / \mathrm{N}$ ratio. 
In fact, the relevant parameter is not so much the absolute flux, but rather the spectroscopic $\mathrm{S} / \mathrm{N}$ ratio, and even more the impact of this spectroscopic $\mathrm{S} / \mathrm{N}$ on the reconstruction of the velocity field. One has to choose a parameter which is supposed to vary in a monotonic and objective way with this impact, establishing eventually the relationship between both. As far as I can think of now, such a parameter could be 'coupling $\times$ D' (more relevant, I believe, than 'coupling times $D^{2}$ ').

Crampton: You didn't mention the really difficult aspect of MOAO: MEMS DMS with adequate size and stroke, open-loop control and adequate computing power to control 30 AO systems with data from $>5$ LGS and $>5$ NGS.

Bouchet: You are perfectly right, the open-loop control is clearly the issue. However, it seems that we can now find on the market DMs which should be suited for MOAO applications. We are now at Observatiore de Paris (in collaboration with Laboratoire d'Astrophysique de Marseille) engaged in a series of tests to check the feasibility of MOAO with some of such DMs. A special optical bench (SESAME) has been built for that purpose. The first results are indeed very encouraging. 